\title{
BMJ Open Efficacy of periacetabular osteotomy followed by progressive resistance training compared to progressive resistance training as non-surgical treatment in patients with hip dysplasia (PreserveHip) - a protocol for a randomised controlled trial
}

Lisa Cecilie Urup Reimer (D) , ${ }^{1}$ Stig Storgaard Jakobsen, ${ }^{1}$ Louise Mortensen, ${ }^{1}$ Ulrik Dalgas, ${ }^{2}$ Julie Sandell Jacobsen, ${ }^{3}$ Kjeld Soballe, ${ }^{1}$ Tone Bere, ${ }^{4}$ Jan Erik Madsen, ${ }^{4}$ Lars Nordsletten, ${ }^{4,5}$ May Arna Risberg, ${ }^{4}$ Inger Mechlenburg ${ }^{1,6}$

To cite: Reimer LCU, Jakobsen SS, Mortensen L, et al. Efficacy of periacetabular osteotomy followed by progressive resistance training compared to progressive resistance training as nonsurgical treatment in patients with hip dysplasia (PreserveHip) - a protocol for a randomised controlled trial. BMJ Open 2019;9:e032782. doi:10.1136/ bmjopen-2019-032782

- Prepublication history for this paper is available online. To view these files, please visit the journal online (http://dx.doi. org/10.1136/bmjopen-2019032782).

Received 05 July 2019 Revised 11 November 2019 Accepted 20 November 2019

Check for updates

(C) Author(s) (or their employer(s)) 2019. Re-use permitted under CC BY-NC. No commercial re-use. See rights and permissions. Published by BMJ.

For numbered affiliations see end of article.

Correspondence to Dr Lisa Cecilie Urup Reimer; lisareimer@clin.au.dk

\section{ABSTRACT}

Introduction Periacetabular osteotomy (PAO) is an established treatment for adolescent and adult patients with hip dysplasia. However, the efficacy of PAO has not been tested against another surgical intervention or conservative treatment in a randomised controlled trial before. We suggest that progressive resistance training (PRT) could be an alternative to PA0. The primary aim of this trial is therefore to examine the efficacy of PAO followed by 4 months of usual care followed by 8 months of PRT compared to 12 months of solely PRT in patients with hip dysplasia eligible for PAO in terms of patientreported pain measured by The Copenhagen Hip and Groin Outcome Score (HAGOS).

Methods and analysis This trial is a single-blinded multicentre randomised controlled clinical trial, where patients with hip dysplasia, who are eligible for PA0, will be randomised to either PAO followed by usual care and PRT or PRT only. Primary outcome is patient-reported pain, measured on the subscale pain on the HAGOS questionnaire 12 months after initiation of PAO or PRT. The key secondary outcomes are the other subscales of the HAGOS, adverse and serious adverse events, usage of painkillers (yes/no) and type of analgesics. Based on the sample size calculation, the trial needs to include 96 patients.

Ethics and dissemination The trial is approved by the Central Denmark Region Committee on Biomedical Research Ethics (Journal No 1-10-72-234-18) and by the Danish Data Protection Agency (Journal No 1-16-02-12019). The trial is also approved by The Regional Committee for Medical and Health Research Ethics, Region South-East Norway (Ref. 2018/1603). All results from this trial will be published in international peer-reviewed scientific journals regardless of whether the results are positive, negative or inconclusive.

Trial registration number NCT03941171

\section{Strengths and limitations of this study}

This is the first head-to-head comparison to evaluate the additive effect of periacetabular osteotomy (PA0) in addition to non-surgical treatment in patients with hip dysplasia scheduled for PAO.

- The trial will provide valuable evidence to surgeons, physiotherapists and decision-makers by highlighting the efficacy, benefits and harms of the surgical and non-surgical treatment approach, respectively.

- The results are expected to have an immediate substantial impact on clinical practise by providing new evidence to achieve optimal allocation of health care resources as well as markedly improved knowledge when informing patients with hip dysplasia about their options.

- The trial is a multicentre randomised controlled and assessor blinded trial, conducted at two sites in two countries.

- A limitation is that the patients cannot be blinded towards the intervention.

\section{INTRODUCTION}

Hip dysplasia is associated with development of early osteoarthritis (OA). ${ }^{1-3}$ However, not everyone with radiologically verified hip dysplasia develops OA. Periacetabular osteotomy $(\mathrm{PAO})^{45}$ is an established treatment for hip dysplasia in adolescents and adults. ${ }^{6-9}$ The aim of PAO is to improve pain and prevent secondary OA by improvement in the hip biomechanics. ${ }^{10}$ However, studies describing the natural history of hip dysplasia are lacking. The lack of knowledge is problematic since patients are offered a surgery 
with potential complications mainly based on pain indication without knowing if $\mathrm{OA}$ would progress. In a longitudinal trial, 136 controls were compared with 81 persons with mild or moderate radiological verified hip dysplasia. ${ }^{11}$ The participants were followed for a decade, but the results of the study did not document a tendency for radiological hip degeneration. In contrast, Morita et $a l^{12}$ found that the probability of OA progression was $13 \%$ in a cohort of 88 patients with hip dysplasia who had received a rotational acetabular osteotomy in the contralateral hip 20 years earlier.

Patients with hip dysplasia typically experience hip pain and reduced walking distance. The pain is localised to the groin area and can be sharp, sudden and sometimes radiate towards the knee. ${ }^{13}$ This results in reduced patient-reported and performance-based physical function. ${ }^{78} 14-16$ In addition, Sørensen et al ${ }^{14}$ showed that patients with hip dysplasia had weaker hip flexor and abductor muscles than age and gender matched controls. Only few trials have investigated physical training for this patient group. ${ }^{17-19}$ Importantly, trials in hip $\mathrm{OA}^{20}{ }^{21}$ have shown, that progressive resistance training (PRT) seems to be a promising exercise modality that may relieve pain and improve function. To our knowledge, no trials have applied PRT in hip dysplasia. We therefore performed a pilot PRT trial ${ }^{15}$ on 17 patients with hip dysplasia scheduled for PAO and found that PRT is feasible with few and minor adverse events and that the patients were motivated for the training (high training compliance). The pilot trial further indicated decreased patient-reported pain, symptoms and better patient-reported scores for sport and recreation measured by The Copenhagen Hip and Groin Outcome Score (HAGOS). Moreover, we found increased performance-based function and increased hip flexion muscle strength on the affected side. As such, a well-powered randomisedcontrolled trial (RCT) comparing PRT to PAO in hip dysplasia seems justified.

In further support of the aforementioned RCT, patients, their relatives, healthcare providers and decision-makers have a common interest in investigating the efficacy of PAO. As described by Wartolowska et $a l,{ }^{22}$ it is reasonable to assume that surgery is associated with a placebo effect. First, because invasive procedures have a stronger placebo effect than non-surgical ones and, second, because a confident diagnosis and a decisive approach to treatment, typical for surgery, usually results in a strong placebo effect. ${ }^{22}$ A recent survey ${ }^{23}$ among British shoulder surgeons showed that surgeons generally agreed that a placebo component to surgical intervention might exist. With the increased use of PAO worldwide and expanded indications for PAO, such as acetabular retroversion and femoroacetabular impingement syndrome, ${ }^{162425}$ it is problematic that the efficacy of PAO has not been investigated in a randomised controlled trial.

\section{Aim and hypothesis of the trial}

The primary aim of this trial is to examine the efficacy of PAO followed by 4 months of usual care followed by 8 months of PRT compared to 12 months of a PRT only, in patients with hip dysplasia eligible for PAO, in terms of patient-reported pain measured by HAGOS. Secondary aims are to investigate changes in patient-reported symptoms, physical function in daily living, physical function in sport and recreation, hip and/or groin-related quality of life, generic health status, performance-based function, hip muscle strength, physical activity and adverse events between PAO followed by usual care and PRT compared to PRT only. We hypothesise that PAO followed by usual care and PRT results in significantly less pain at 12 months follow-up compared to PRT only.

\section{MATERIAL \\ Design}

This trial is a multicentre randomised controlled and assessor blinded trial, following the Consolidated Standards of Reporting Trials (CONSORT) guidelines. ${ }^{26}$ Change in primary outcome will be measured from baseline to 12 months follow-up, while change in secondary outcomes will be measured from baseline to 4 and 12 months follow-up. In addition, 5 year and 10 year follow-up with questionnaires is planned.

\section{Patients}

\section{Setting and location}

Patients will be recruited from the Departments of Orthopaedic Surgery at Aarhus University Hospital, Denmark, and at Division of Orthopaedic Surgery at Oslo University Hospital, Norway. Approximately 130 PAOs are performed yearly in Aarhus, and $40 \mathrm{PAO}$ are performed in Oslo yearly. We expect to recruit 96 patients. Both centres will be including patients, but the analysis will be performed at Aarhus University Hospital.

\section{Eligibility criteria}

1. Patients aged 18 to 40 years and diagnosed with hip dysplasia referred from primary care to the Department of Orthopaedic Surgery at one of the two participating hospitals.

2. Considered eligible for PAO by a surgeon.

3. Radiographically verified hip dysplasia (Wiberg's centre-edge angle $<25$ degrees and Acetabular Index angle $>10$ degrees) and clinical symptoms.

4. Range of motion: internal rotation $>15$ degrees, external rotation $>15$ degrees, hip flexion $>110$ degrees.

5. Able to drive or commute to training sessions.

\section{Exclusion criteria}

1. OA degree $>1$ on classification of Tönnis.

2. CE-angle $<10$ degrees.

3. Retroverted acetabulum (crossover sign and posterior wall sign).

4. Previous pelvic surgery for hip dysplasia (affected side).

5. Legg-Calvé-Perthes or epiphysiolysis.

6. Simultaneous bilateral PAO. 
7. Previous surgery for herniated disc, spondylodesis, arthroplasty of hip, knee or ankle.

8. Previous surgery of the hip (tenotomy of iliopsoas tendon, z-plastic of the iliotibial tract or hip arthroscopy) in index leg.

9. Neurological or rheumatoid diseases that affect the hip function.

10. Inadequacy in written and spoken Danish or Norwegian.

11. Body Mass Index $>25$.

\section{Randomisation}

After baseline assessment, the patients will be randomised in a 1:1 ratio to either PAO followed by usual care and PRT (PAO-group) or PRT only (PRT-group). A computergenerated list of random numbers will be set up in the Research Electronic Data Capture (REDCap) randomise tool. Administrators of the randomisation procedure will be blinded to block sizes and randomisation sequence at all times during the trial period. Allocation concealment will be ensured, as the randomisation will not be performed and revealed before the patient has been irreversibly included in the trial. After randomisation a secretary or project coordinator, who is otherwise not affiliated with the trial, will refer patients to surgery or to the treating physiotherapist/physiotherapy student who contacts the patient for an appointment of the first exercise session.

PRT-group

The PRT-group receives 4 months of supervised PRT two times per week. A physiotherapist or physiotherapist student will supervise all training sessions the first 4 weeks.
The following 4 weeks, six out of eight training sessions are supervised and from week 9 to 16 , half of the training sessions ( 8 out of 16) are supervised. After these 4 months ( 16 weeks), patients receive a free membership to a fitness centre near their home address and are encouraged to train on their own two times per week until 12 months follow-up with one supervised session per month. Supervised training sessions will be conducted at VIA University College (in Denmark) or at a physiotherapy practice (in Norway). If the included patients randomised to the PRTgroup do not find they benefit from the PRT they can crossover from 4 months (see figure 1 and the section "Anchor question"), or at any time later throughout the intervention. Four months is the normal time to wait when being on the waiting list for a PAO surgery, but if patients wish to crossover after 4 months of PRT, they will not be put on a new waiting list but directly scheduled for PAO.

\section{PA0-group}

$\mathrm{PAO}$ will be performed with the transsartorial approach ${ }^{5}$ or the anterior pelvic approach. ${ }^{4} \mathrm{X}$-rays (AP pelvic and AP hip) will be performed after 6 weeks, 4 months, 12 months, 5 years and 10 years. Patients commence postoperative rehabilitation as usual until 4 months after the operation. Usual care means that the patients follow a rehabilitation programme guided by a physiotherapist specialised in hip problems, with focus on stability and strength after the operation, as well as regaining a normal gait pattern. The physiotherapist will adapt the postoperative rehabilitation to the patients need and thus usual

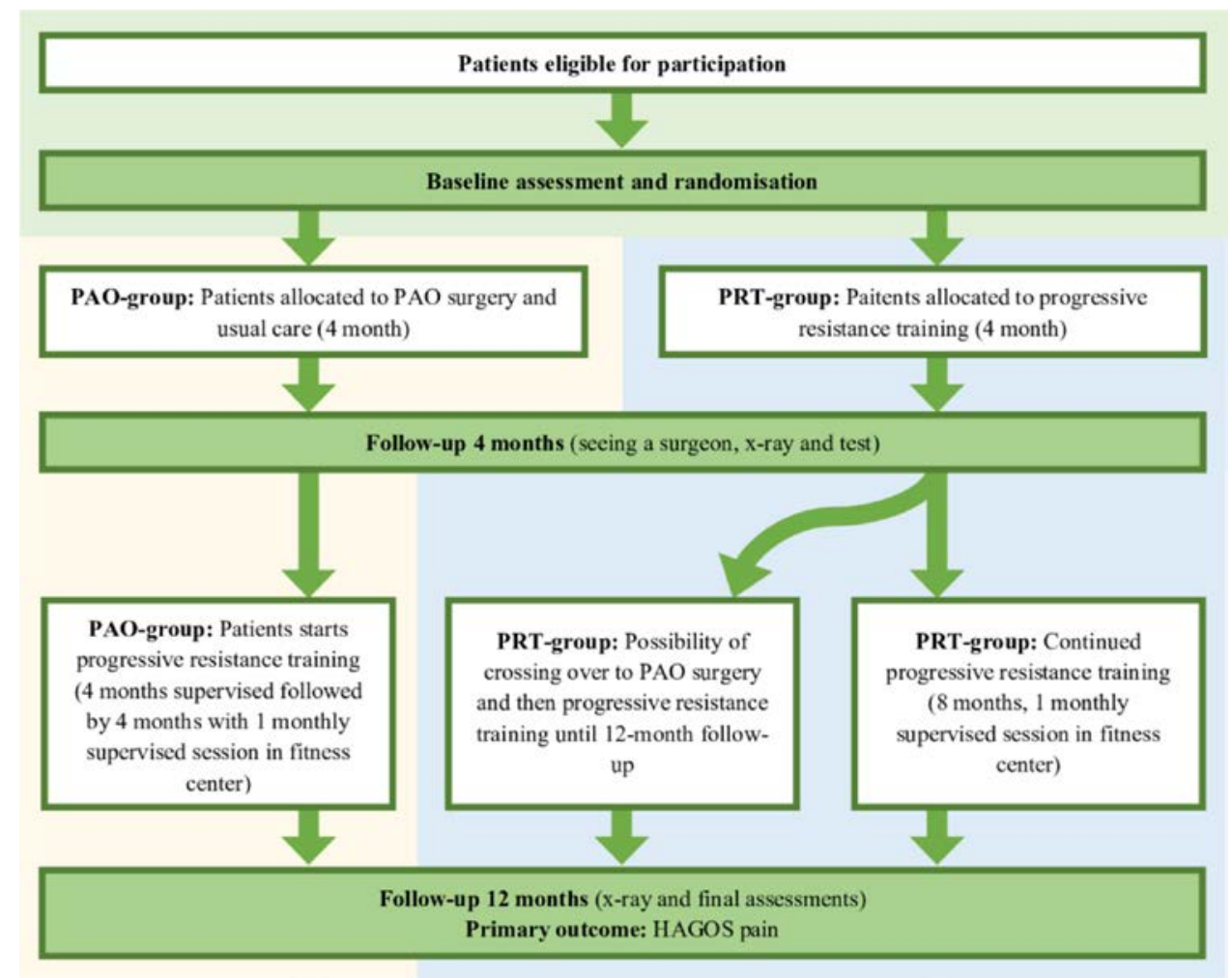

Figure 1 Patient flow through the trial. 
care will differ between patients. Four months postoperative the patients complete usual care commence with the same PRT intervention programme as the PRT group, until 12 months follow-up (see description above).

\section{METHOD}

\section{Training program}

The PRT programme involves $10 \mathrm{~min}$ of warm up followed by four exercises including sets of loaded squats, hip extension, hip flexion and hip abduction. Loaded squat is performed standing with a barbell or dumbbells and target hip- and knee- extensors and flexors. Hip extension is performed standing in a cable-tower with a cable fixed around the ankle and the leg is moved backward and upward in a stretched position to perform hipextension against resistance. Hip flexion is performed standing in a cable-tower with the cable fixed around the ankle and the knee is moved forward and upward to perform hip-flexion against resistance. Hip abduction is performed standing in a cable-tower with a cable fixed around the leg and leg is moved out to the side and up while kept stretched, to perform hip-abduction against resistance. To avoid muscle soreness of the affected leg (defined as index leg), squat is performed before the unilateral exercises. After week 16, all exercises are performed by both legs to train stability for the index leg and because the majority of the patients have bilateral hip dysplasia and hence probably profit from bilateral resistance training. The exercises are focused on strengthening all the muscles around the hip. Since patients with hip dysplasia primarily experience decreased strength in hip flexors and hip abductors, ${ }^{27}$ these muscles are incorporated in the training programme, but to assure a symmetrical strengthening of the hip muscles, hip extensors are also included in the training programme.
Only four exercises have been included in the training programme to ensure that the training sessions do not exceed $60 \mathrm{~min}$, and consequently patients are more likely to adhere to the training programme. The exercises are simple to perform, and all equipment used are standard equipment in essentially all fitness centres. The absolute training load will be individually adjusted on a set-by-set basis, using the plus two principle (if the patient is able to perform two or more repetitions than required, the load is increased). Hip related pain levels up to 5 on the Visual Analogue Scale (VAS) is considered acceptable during exercise. ${ }^{11}$ Progression of relative load will be performed as described in table 1.

\section{Outcomes}

Outcome assessments will be performed at baseline, and at 4 months and 12 months follow-up (after initiation of surgical/non-surgical treatment). An assessor blinded to group allocation will conduct baseline and follow-up measurements. The patients will be contacted and asked to complete hip-related questionnaires, 5 and10 yearsafter inclusion into the trial. An overview of the different outcomes is presented in table 2.

\section{Primary outcome}

The pain subscale of the patient-reported questionnaire HAGOS, were the total score ranges from 0 (worst) to 100 (best) ${ }^{27}$ HAGOS is a valid, reliable and responsive patient-reported outcome in young patients with hip and groin related pain. ${ }^{27} \mathrm{~A}$ minimal clinically relevant difference of the HAGOS pain subscale is considered to be 9.7. ${ }^{28}$

\section{Secondary outcome}

The most important secondary outcomes are presented as key secondary outcomes. The key secondary outcomes

\begin{tabular}{|c|c|c|c|c|}
\hline Exercise variable & Week 1-2 & Week 3-4 & Week 5-6 & Week 7-52 \\
\hline Load & $15 \mathrm{RM}$ & $12 \mathrm{RM}$ & $10 \mathrm{RM}$ & $8 \mathrm{RM}$ \\
\hline Repetitions & 10 & 12 & 10 & 8 \\
\hline Set per session & 3 & 3 & 4 & 4 \\
\hline Rest between sets & $90 \mathrm{~s}$ & $90 \mathrm{~s}$ & $120 \mathrm{~s}$ & $120 \mathrm{~s}$ \\
\hline Sessions per week & 2 & 2 & 2 & 2 \\
\hline Duration of training period & 52 weeks & 52 weeks & 52 weeks & 52 weeks \\
\hline \multirow[t]{4}{*}{ Exercises } & Loaded squat & Loaded squat & Loaded squat & Loaded squat \\
\hline & Hip extension & Hip extension & Hip extension & Hip extension \\
\hline & Hip flexion & Hip flexion & Hip flexion & Hip flexion \\
\hline & Hip abduction & Hip abduction & Hip abduction & Hip abduction \\
\hline Contraction failure in each set & Yes & Yes & Yes & Yes \\
\hline Range of motion & Maximal possible & Maximal possible & Maximal possible & Maximal possible \\
\hline Rest between training sessions & $>36$ hours & $>36$ hours & $>36$ hours & $>36$ hours \\
\hline
\end{tabular}

After week 16, all exercises are performed by both legs.

$\mathrm{RM}$, repetition maximum. 
Table 2 Assessments and procedures

\begin{tabular}{|c|c|c|c|c|c|c|}
\hline & Baseline & Surgery & 4 months & 12 months & 5 years & 10 years \\
\hline \multicolumn{7}{|l|}{ Baseline characteristics } \\
\hline Gender & $\mathrm{X}$ & & & & & \\
\hline Height & $\mathrm{X}$ & & & & & \\
\hline Weight & 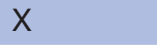 & & $x$ & $\mathrm{X}$ & & \\
\hline Marital status & $x$ & & & & & \\
\hline Educational level & $x$ & & & & & \\
\hline Employment status & $x$ & & & & & \\
\hline Physical activity and exercise & $x$ & & $x$ & $x$ & & \\
\hline Alcohol intake & $\mathrm{x}$ & & & & & \\
\hline
\end{tabular}

\section{Patient-reported outcomes}

\begin{tabular}{|c|c|c|c|c|c|}
\hline HAGOS & $\mathrm{X}$ & $X$ & $X$ & 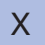 & $X$ \\
\hline EQ-5D-5L & $X$ & $X$ & $\mathrm{X}$ & $x$ & $\mathrm{X}$ \\
\hline FJS-12 & $x$ & $x$ & $\mathrm{X}$ & $x$ & $\mathrm{X}$ \\
\hline \multicolumn{6}{|l|}{ Physical performance tests } \\
\hline Single leg hop for distance & $X$ & $\mathrm{X}$ & $\mathrm{X}$ & & \\
\hline Isometric hip muscle strength* & $x$ & $\mathrm{X}$ & $\mathrm{X}$ & & \\
\hline \multicolumn{6}{|l|}{ Physical activity } \\
\hline Tri-axial accelerometry & $X$ & & $\mathrm{X}$ & & \\
\hline \multicolumn{6}{|l|}{ Treatment related variables } \\
\hline X-ray & $X$ & $X$ & $\mathrm{X}$ & $X$ & $\mathrm{X}$ \\
\hline Visual Analogue Scaleł & $X$ & $\mathrm{X}$ & $\mathrm{X}$ & & \\
\hline Other treatments & & $x$ & $\mathrm{X}$ & & \\
\hline Usage of analgesics & $X$ & $X$ & $\mathrm{X}$ & & \\
\hline Delay to surgery (only PRT-group) & & & & $x$ & $x$ \\
\hline Surgery (only PRT-group) & & $\mathrm{X}$ & $X$ & $X$ & $X$ \\
\hline
\end{tabular}

*Isometric hip muscle strength: hip flexion, extension and abduction.

tSee box 1

¥VAS scores will be obtained before and after training.

EQ-5D-5L, European Quality of life 5 Dimensions with 5 Levels; FJS-12, Forgotten Joint Score-12; HAGOS, The Copenhagen Hip and Groin Outcome Score; PRT, progressive resistance training; VAS, Visual Analogue Scale.

are the other subscales of the HAGOS covering Symptoms, Physical function in daily living, Physical function in Sport and Recreation and Quality of Life; Single leg hop for distance $;^{29}{ }^{30}$ adverse and serious adverse events (see box 1). ${ }^{31}$ Usage of painkillers (yes/no) and type of analgesics is also part of the key secondary outcomes. The other secondary outcomes are; HAGOS subscale Participation in Physical Activities, pain reported by the VAS; ${ }^{32}$
Forgotten Joint Score-12; ${ }^{33}$ Y-balance test ${ }^{34}$ and isometric measured hip muscle strength (flexion, extension and abduction).

\section{Exploratory outcomes}

Tri-axial accelerometer (only at baseline and 12 months follow-up); EuropeanQuality of life 5 Dimensions with 5 Levels (EQ-5D-5L) $;^{35}$ delay to surgery and demographic 


\section{Box 1 Adverse and serious adverse events}

Adverse events
Haematoma
Delayed wound closure
Dysaesthesia of lateral femoral cutaneous nerve
Malpositioning; retroversion or insufficient reorientation. Insufficient re-
orientation (coverage) - optimally is the CE-angle 30 to 40 degrees and
the Al-angle 0 to 10 degrees.
Heterotopic ossifications (Brooker I and II)
Urinary tract infections
Infection not requiring surgical revision
Serious adverse events
Avascular necrosis of the femoral head or acetabulum
Nerve palsy
Major bleeding (administration of more than five blood units intraoper-
atively and postoperatively)
Peroneal and femoral neurapraxia
Deep vein thrombosis
Pulmonary embolism
Stress fracture of ischial bone and posterior column
Intraarticular osteotomy
Heterotopic ossifications (Brooker III and IV)
Infection requiring surgical revision
Loss of fixation/loss of reorientation
Delayed or non-union of pubic, ischial or iliac bone

differences between crossover patients compared with patients as treated in the PRT group.

\section{Demographic data}

Gender, age, height, weight, duration of hip symptoms, civil status, educational level, employment status, physical activity and exercise, alcohol intake, smoking behaviours and comorbidities.

\section{Assessment of compliance}

Compliance to training will be registered from the patients' training protocols, described as number of sessions attended versus number of planned sessions according to the protocol in per cent. Compliance to training will be calculated both for those who complete the intervention and for all patients, including drop-outs. High compliance is defined as $\geq 70 \%$ attendance to the supervised sessions the first 4 months. Number of selftraining sessions will be recorded in a training diary and high compliance to self-training (from 4 to 12 months follow-up) is defined as attendance to the PRT of $\geq 50 \%$.

\section{X-rays}

X-rays will be performed with the patient in standing position (AP pelvic) and in supine position (AP hip), at baseline and at every follow-up for both groups. X-rays will also be repeated at 6 weeks for the PAO-group as part of the standard postoperative care.

\section{Data entry}

In Denmark the software REDCap will be used for data entering, while EpiData will be used in Norway.

\section{Anchor question}

After 4 months, the patients will have an appointment with the surgeon. Before this meeting the patients will fill out the anchor question (described below) and the HAGOS questionnaire. These two questionnaires will form the basis of the talk with the surgeon. For patients allocated to the PRT-group, the surgeon will ask the patient to evaluate to which extent the a priori hip problems have been addressed, and the patient and surgeon decide whether the patient continues in the PRT-group they have been randomised to or is crossing over to PAO. The decision of crossing over is thus a decision made between the surgeon and the patient, based on the anchor question and the HAGOS questionnaire. After talking to the surgeon, function and muscle strength will be tested.

\section{Anchor question}

PAO-group: How is your operated hip now compared with before surgery? Much better, slightly better, the same, slightly worse or much worse?

PRT-group: How is your hip now compared with before you started this training programme? Much better, slightly better, the same, slightly worse or much worse?

\section{Sample size}

A minimal clinically relevant difference of the HAGOS pain subscale is considered to be 9.7 (28). Based on a previous pilot trial the SD of HAGOS pain in $\mathrm{PAO}$ patients is 16.2 (15). Given a power of 0.80 and two-sided significance level $\alpha=0.05$, the estimated sample size of each intervention group is 44 patients. Allowing for possible crossovers and loss to follow-up, the number of included patients in each intervention group will be 48 patients.

\section{Data availability statement}

Aarhus University Hospital is responsible for handling all personal data provided by both sites in accordance to the Clinical Trial Agreement and the EU General Data Protection Regulation. Oslo University Hospital agree that information directly related to the protocol and trial, including data, material, Intellectual Property and results generated from the trial shall be the property of Aarhus University Hospital, and shall be treated in strict confidence, and shall not be disclosed to any third party, or use for its benefit or the benefit of any third party, without the prior written consent of Aarhus University Hospital, except for data that is (i) publicly known or available from other sources who are not under a confidentially obligation to the other party, (ii) has been made available by the other party without confidentiality obligation, (iii) is independently developed or otherwise already known by or available to the other party without a confidentiality obligation or (iv) is already required disclosed by law.

\section{Statistical considerations}

All descriptive statistics and tests will be reported in accordance with the recommendations of the 'Enhancing the QUAlity and Transparency Of health Research' (EQUATOR) ${ }^{36}$ network and the CONSORT statement. ${ }^{26}$ The primary 
efficacy analysis will be assessment of the between-group difference in change in the HAGOS pain subscale from baseline to 12 months after initiating the treatment (primary end-point). The primary analysis will follow the intention-to-treat principle and a mixed effects model will be used. Sensitivity and exploratory analysis will be performed with the purposes to test the robustness of the results per-protocol with good compliance (defined as participation in $\geq 70 \%$ of the training sessions) and as-treated analysis, in which patients will be analysed based on their adherence to the randomised treatment expecting three groups ${ }^{1}$ : patients randomised to $\mathrm{PAO},{ }^{2}$ patients randomised to PRT without undergoing PAO in the follow-up period, ${ }^{3}$ patients randomised to PRT undergoing PAO in the follow-up period.

\section{Ethics and dissemination}

Before inclusion, all patients will have to give their written, informed consent in accordance with the Declaration of Helsinki II. All data and information collected in regard to this trial will be treated confidentially by the researchers and staff connected to the trial.

\section{Patient and public involvement}

During the development of the trial design, we have interviewed a group of patients with hip dysplasia with the purpose of gaining knowledge on the patients' thoughts on participating in a clinical trial that investigates the efficacy of joint preserving surgery compared with a PRT programme. The patients were asked to consider how often they would be able to train, how far they would be willing to commute to the training facility and what their primary reason for seeking treatment had been. Likewise, they were asked about what was most important for them to achieve with an operation or a training intervention. This was performed in order to use the obtained knowledge to improve our patient information, the method of patient recruitment and the PRT programme.

\section{DISCUSSION}

This is the first head-to-head comparison to evaluate the additive effect of PAO in addition to non-surgical treatment in patients with hip dysplasia scheduled for PAO. The trial will provide valuable evidence to surgeons, physiotherapists and decision-makers by highlighting the efficacy, benefits and harms of the surgical and nonsurgical treatment approach, respectively. The results are expected to have an immediate substantial impact on clinical practise.

Since the trial is designed to be an assessor blinded randomised controlled trial, it reaches the highest evidence level. For obviously reasons it is not possible to blind the patients towards the intervention, which is a limitation of the trial. The trial is conducted at two University Hospitals and the patients are regular patients, thus the infrastructure used is of high standard. Both hospitals have specific hip units and have all necessary hospital equipment available including operational environment and postoperative hospitalisation. All outcomes are valid and reliable outcome measures and consist of both multiple patient-reported outcomes and objective outcome measures.

There can be unforeseen risks in connection with all trials, but these are considered minimal in this trail. When performing PRT, it is normal to experience muscle soreness, and based on the experience from our earlier feasibility trial ${ }^{15}$ testing PRT in patients with hip dysplasia, we know that there are times where the patients can experience muscle-related pain. The patients are thus asked to score their pain before and after each training session, to ensure that the training does not aggravate the hip pain. All methods included in this trial have been used in previous approved trials.

All results from the trial will be published in international peer-reviewed scientific journals regardless of whether the results are positive, negative or inconclusive.

\section{Author affiliations}

${ }^{1}$ Orthopedic Surgery, Aarhus University Hospital, Aarhus, Denmark

${ }^{2}$ Public Health - Sport, Aarhus University, Aarhus, Denmark

${ }^{3}$ Physiotherapy \& Research Centre for Health and Welfare Technology, Programme

for Rehabilitation, VIA University College, Aarhus, Denmark

${ }^{4}$ Orthopedic Surgery, Oslo University Hospital, Oslo, Norway

${ }^{5}$ Faculty of Medicine, University of Oslo, Oslo, Norway

${ }^{6}$ Clinical Medicine, Aarhus University, Aarhus, Denmark

Contributors LR, SJ, LM, UD, JJ, KS, TB, JM, LN, MR and IM were a part of designing the trial and approved the final version of the protocol. LR and IM wrote the protocol and SJ, LM, UD, JJ, KS, TB, JM, LN and MR revised the protocol.

Funding This trial is supported by Aarhus University ( $550000 \mathrm{dkk}$ ), Aase and Ejnar Danielsens Foundation (84 000 dkk), Alfred Benzons Foundation (1 164000 dkk), Andelsforeningernes Humanitære og Kulturelle Fond (20 000 dkk), Fondsstiftelsen ved Oslo Universitetssykehus (148.000 nok), The Danish Rheumatism Association (673 000 dkk), The Family Hede Nielsens Foundation (10 000 dkk), The FrimodtHeineke Foundation (15 $000 \mathrm{dkk}$ ) The Norwegian Fund for Post-Graduate Training in Physiotherapy (252.000 nok), The Riisfort Foundation (104 $800 \mathrm{dkk}$ ) and Vanførefonden (100 $000 \mathrm{dkk}$ ). The foundations had no role in planning the trial and did only deliver financial support.

Competing interests None declared.

Patient consent for publication Not required.

Ethics approval The Central Denmark Region Committee on Biomedical Research Ethics (Journal No 1-10-72-234-18), the Danish Data Protection Agency (Journal No 1-16-02-120-19) and The Regional Committee for Medical and Health Research Ethics, Region South-East Norway (Ref. 2018/1603) approved the trial.

Provenance and peer review Not commissioned; externally peer reviewed.

Data availability statement Data are available upon reasonable request. After publishing, data regarding Danish patients will be stored at The Danish National Archives, while the data regarding Norwegian patients will be stored at The Norwegian National Archives. With the right approvals, data can be accessed there.

Open access This is an open access article distributed in accordance with the Creative Commons Attribution Non Commercial (CC BY-NC 4.0) license, which permits others to distribute, remix, adapt, build upon this work noncommercially, and license their derivative works on different terms, provided the original work is properly cited, appropriate credit is given, any changes made indicated, and the use is non-commercial. See: http://creativecommons.org/ licenses/by-nc/4.0/.

\section{ORCID iD}

Lisa Cecilie Urup Reimer http://orcid.org/0000-0003-4666-4622 


\section{REFERENCES}

1 Murphy SB, Ganz R, Müller ME. The prognosis in untreated dysplasia of the hip. A study of radiographic factors that predict the outcome. J Bone Joint Surg Am 1995;77:985-9.

2 Jacobsen S, Sonne-Holm S. Hip dysplasia: a significant risk factor for the development of hip osteoarthritis. A cross-sectional survey. Rheumatology 2005;44:211-8.

3 Wyles CC, Heidenreich MJ, Jeng J, et al. The John Charnley Award: redefining the natural history of osteoarthritis in patients with hip dysplasia and impingement. Clin Orthop Relat Res 2017;475:336-50.

4 Ganz R, Klaue K, Vinh TS, et al. A new periacetabular osteotomy for the treatment of hip dysplasias. Technique and preliminary results. Clin Orthop Relat Res 1988:26-36.

5 Troelsen A, Elmengaard B, Søballe K. A new minimally invasive Transsartorial approach for Periacetabular osteotomy. J Bone Joint Surg Am 2008;90:493-8.

6 Steppacher SD, Tannast M, Ganz R, et al. Mean 20-year followup of Bernese Periacetabular osteotomy. Clin Orthop Relat Res 2008;466:1633-44.

7 Lerch TD, Steppacher SD, Liechti EF, et al. One-Third of hips after Periacetabular osteotomy survive 30 years with good clinical results, no progression of arthritis, or conversion to THA. Clin Orthop Relat Res 2017;475:1154-68.

8 Hartig-Andreasen C, Troelsen A, Thillemann TM, et al. What factors predict failure 4 to 12 years after Periacetabular osteotomy? Clin Orthop Relat Res 2012;470:2978-87.

9 Mechlenburg I, Nyengaard JR, Gelineck J, et al. Cartilage thickness and cyst volume are unchanged 10 years after Periacetabular osteotomy in patients without hip symptoms. Clin Orthop Relat Res 2015;473:2644-9.

10 Mechlenburg I, Nyengaard J, Rømer L, et al. Changes in load-bearing area after Ganz periacetabular osteotomy evaluated by multislice CT scanning and stereology. Acta Orthop Scand 2004;75:147-53.

11 Jacobsen S, Sonne-Holm S, Søballe K, et al. Joint space width in dysplasia of the hip: a case-control study of 81 adults followed for ten years. J Bone Joint Surg Br 2005;87:471-7.

12 Morita D, Hasegawa Y, Seki T, et al. A possible new radiographic predictor of progression of osteoarthritis in developmental dysplasia of the hip. Clin Orthop Relat Res 2018;476:2157-66.

13 Troelsen A, Rømer L, Søballe K. [Hip dysplasia: clinical assessment, radiologic evaluation and reference]. Ugeskr Laeger 2007;169:394-6.

14 Sørensen H, Nielsen DB, Jacobsen JS, et al. Isokinetic dynamometry and gait analysis reveal different hip joint status in patients with hip dysplasia. Hip Int 2019;29:215-21.

15 Mortensen L, Schultz J, Elsner A, et al. Progressive resistance training in patients with hip dysplasia: a feasibility study. J Rehabil Med 2018:50:751-8.

16 Clohisy JC, Nepple JJ, Ross JR, et al. Does surgical hip dislocation and Periacetabular osteotomy improve pain in patients with Pertheslike deformities and acetabular dysplasia? Clin Orthop Relat Res 2015;473:1370-7.

17 Kanai A, Kiyama T, Genda E, et al. Biomechanical investigation of ambulatory training in patients with acetabular dysplasia. Gait Posture 2008;28:52-7.

18 Kuroda D, Maeyama A, Naito M, et al. Dynamic hip stability, strength and pain before and after hip abductor strengthening exercises for patients with dysplastic hips. Isokinet Exerc Sci 2013;21:95-100.

19 Harris-Hayes M, Czuppon S, Van Dillen LR, et al. Movement-Pattern training to improve function in people with chronic hip joint pain: a feasibility randomized clinical trial. J Orthop Sports Phys Ther 2016;46:452-61.
20 Bieler T, Siersma V, Magnusson SP, et al. In hip osteoarthritis, Nordic walking is superior to strength training and home-based exercise for improving function. Scand J Med Sci Sports 2017;27:873-86.

21 Hermann A, Holsgaard-Larsen A, Zerahn B, et al. Preoperative progressive explosive-type resistance training is feasible and effective in patients with hip osteoarthritis scheduled for total hip arthroplasty - a randomized controlled trial. Osteoarthritis and Cartilage 2016;24:91-8.

22 Wartolowska K, Judge A, Hopewell S, et al. Use of placebo controls in the evaluation of surgery: systematic review. BMJ 2014;348:g3253.

23 Wartolowska K, Beard DJ, Carr AJ. Attitudes and beliefs about placebo surgery among orthopedic shoulder surgeons in the United Kingdom. PLoS One 2014;9:e91699.

24 Zurmühle CA, Anwander H, Albers CE, et al. Periacetabular osteotomy provides higher survivorship than rim trimming for acetabular Retroversion. Clin Orthop Relat Res 2017;475:1138-50.

25 Belzile EL, Beaulé PE, Ryu J-J, et al. Outcomes of joint preservation surgery: comparison of patients with developmental dysplasia of the hip and femoroacetabular impingement. J Hip Preserv Surg 2016;3:hnw033-7.

26 Moher D, Hopewell S, Schulz KF, et al. CONSORT 2010 Explanation and Elaboration: updated guidelines for reporting parallel group randomised trials. J Clin Epidemiol 2010;63:e1-37.

27 Thorborg K, Holmich P, Christensen R, et al. The Copenhagen hip and groin outcome score (HAGOS): development and validation according to the COSMIN checklist. $\mathrm{Br} J$ Sports Med 2011;45:478-91.

28 Thomeé R, Jónasson P, Thorborg K, et al. Cross-Cultural adaptation to Swedish and validation of the Copenhagen hip and groin outcome score (HAGOS) for pain, symptoms and physical function in patients with hip and groin disability due to femoroacetabular impingement. Knee Surg Sports Traumatol Arthrosc 2014;22:835-42.

29 Ageberg E, Cronström A. Agreement between test procedures for the single-leg hop for distance and the single-leg mini squat as measures of lower extremity function. BMC Sports Sci Med Rehabil 2018;10:1-7.

30 Kemp JL, Risberg MA, Schache AG, et al. Patients with Chondrolabral pathology have bilateral functional impairments 12 to 24 months after unilateral hip arthroscopy: a cross-sectional study. $J$ Orthop Sports Phys Ther 2016;46:947-56.

31 Biedermann R, Donnan L, Gabriel A, et al. Complications and patient satisfaction after periacetabular pelvic osteotomy. Int Orthop 2008;32:611-7.

32 Hawker GA, Mian S, Kendzerska T, et al. Measures of adult pain: visual analog scale for pain (vas pain), numeric rating scale for pain (NRS pain), McGill pain questionnaire (MPQ), short-form McGill pain questionnaire (SF-MPQ), chronic pain grade scale (CpGs), short Form-36 bodily pain scale (SF. Arthritis Care Res 2011;63:S240-52.

33 Behrend H, Giesinger K, Giesinger JM, et al. The "forgotten joint" as the ultimate goal in joint arthroplasty: validation of a new patientreported outcome measure. J Arthroplasty 2012;27:430-6.

34 Plisky PJ, Gorman PP, Butler RJ, et al. The reliability of an instrumented device for measuring components of the star excursion balance test. N Am J Sports Phys Ther 2009;4:92-9.

35 Herdman M, Gudex C, Lloyd A, et al. Development and preliminary testing of the new five-level version of EQ-5D (EQ-5D-5L). Qual Life Res 2011;20:1727-36.

36 Christensen R, Bliddal H, Henriksen M. Enhancing the reporting and transparency of rheumatology research: a guide to reporting guidelines. Arthritis Res Ther 2013;15:109-4. 